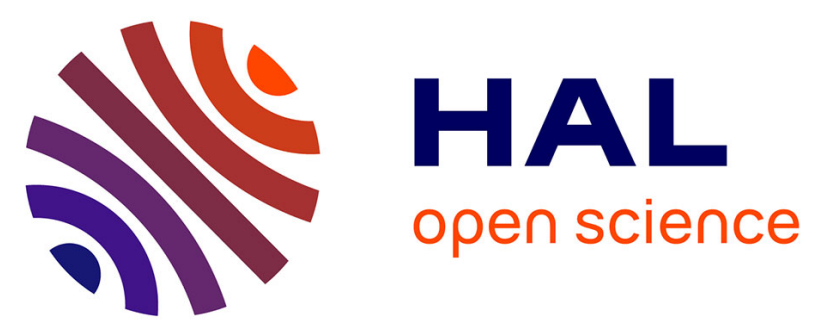

\title{
Proactive user association in wireless small cell networks via collaborative filtering
}

Francesco Pantisano, Mehdi Bennis, Saad Walid, Stefan Valentin, Mérouane Debbah, Alessio Zappone

\section{- To cite this version:}

Francesco Pantisano, Mehdi Bennis, Saad Walid, Stefan Valentin, Mérouane Debbah, et al.. Proactive user association in wireless small cell networks via collaborative filtering. Asilomar 2013, Nov 2013, Pacific Grove, United States. pp.1601 - 1605, 10.1109/ACSSC.2013.6810568 . hal-00925992

\section{HAL Id: hal-00925992 \\ https://hal-centralesupelec.archives-ouvertes.fr/hal-00925992}

Submitted on 8 Jan 2014

HAL is a multi-disciplinary open access archive for the deposit and dissemination of scientific research documents, whether they are published or not. The documents may come from teaching and research institutions in France or abroad, or from public or private research centers.
L'archive ouverte pluridisciplinaire HAL, est destinée au dépôt et à la diffusion de documents scientifiques de niveau recherche, publiés ou non, émanant des établissements d'enseignement et de recherche français ou étrangers, des laboratoires publics ou privés. 


\title{
Proactive User Association in Wireless Small Cell Networks via Collaborative Filtering
}

\author{
Francesco Pantisano, Mehdi Bennis, Walid Saad, Stefan Valentin, Mérouane Debbah, and Alessio Zappone
}

\begin{abstract}
In this paper, we propose a proactive cell association framework for small cell networks, based on content recommendations. We focus on multimedia data services and characterize the user's quality of experience (QoE) in terms of mean opinion scores (MOSs) that accurately reflect characteristics of the wireless transmissions and data applications. Based on such information, we propose a collaborative filtering approach that enables the small base stations (SBSs) to exploit the correlation across similar user and application requirements to predict the QoE that a UE-SBS association delivers. By leveraging such knowledge, the SBSs ultimately devise better-informed user-cell associations and more precise bandwidth allocations. We model this problem as a matching game with externalities, propose a decentralized algorithm based interference graph theory, and discuss the matching stability conditions in the proposed approach. Simulation results show that the proposed solution yields more precise bandwidth allocations reaching up to $19 \%$ spectrum savings, compared to conventional cell association approaches.
\end{abstract}

Index terms: small cell networks; matching games; game theory; proactive bandwidth reservation; context-awareness; quality of experience.

\section{INTRODUCTION}

Meeting the exponentially growing traffic demands of wireless data application in a cost-effective way, mandates a paradigm shift in the design of wireless cellular networks. To this end, small cell base stations (SBSs) (i.e., picocells, femtocells) have emerged as a candidate solution for meeting stringent quality-of-service (QoS) requirements in a scalable and spectrum efficient way [1], [2]. However, even with the latest advancements in transmission techniques, small cell deployments still face a number of fundamental challenges that include the user admission control and the spectral resource management. Most notably, the large number of user equipments (UE) and serving base stations exacerbates the complexity of the UE-SBS association problem for downlink transmission [3]. In fact, the small cell scenario significantly differs from traditional macro-cellular networks due to the density of SBSs, heterogeneity (disparate coverage areas and cell sizes), and unbalanced resource availability [3-5]. These distinct features make macrocell-oriented user association techniques as in [2], [6], [7] unsuitable to the topology of small cell networks, notably when stringent QoS constraints are required.

Dr. Pantisano and Dr. Bennis are with the Centre for Wireless Communications, Oulu, Finland, (email: \{fpantisa,bennis\}@ee.oulu.fi). Prof. Saad (walid@miami.edu) is with Electrical and Computer Engineering Department, University of Miami, Coral Gables, FL, USA. Dr. Valentin (stefan.valentin@alcatel-lucent.com) is with Bell Labs, Alcatel-Lucent, Stuttgart, Germany. Prof. Debbah (merouane.debbah@supelec.fr) is the Alcatel-Lucent Chair in Flexible Radio, SUPÉLEC, Gif-sur-Yvette, France. Dr. Zappone (alessio.zappone@tu-dresden.de) is with Dresden University of Technology. The research leading to this paper has been partly supported by the Celtic-Plus project SHARING ( project number C2012/1-8) and by the U.S. National Science Foundation under Grant CNS-1253731.
One promising approach for addressing the cell association problem is through the exploration of additional context information extracted from the user's devices that can include data such as the typical set of active applications [8]. By exploiting this knowledge, each SBS can proactively allocate the necessary spectral resources and make better informed user associations decisions, based on each UE's quality of experience (QoE) requirements and on the current spectral resource availability. In summary, based on the knowledge of the UEs' data application requirements, SBS networks can anticipate the resource allocation and devise the UE-SBS association that delivers the largest individual QoE.

Anticipating the network operations based on an user context information is an emerging topic in wireless cellular communications. In fact, such a technique - extensively surveyed in [9] - can deliver high QoE, notably when combined with cross-tier optimization techniques. Along this research direction, context information has been exploited for the delivery of popular multimedia contents, by proactively caching file copies with the aim of preventing backhaul congestion [10], [11]. Independently, another line of research has applied predictive algorithms to estimate the data rate requirements of high quality video streaming applications and optimize the spectrum allocation [12]. These examples show remarkable evidence on the benefit of combining context information and predictive algorithms into traditional cellular network operations.

The main contribution of this paper is to combine concepts of QoE prediction and user context for solving the UE-SBS association problem in the downlink of small cell networks. We explore how the typical set of active applications and the qualitative feedback on past data services can be translated, at the network level, into decisions on which UE should be serviced, and by which SBS. We address this problem in two phases. First, we model the QoE feedbacks as mean opinion scores (MOSs), which account for the characteristics of both wireless transmissions and the multimedia data services in use at the UE's side. We assume that each SBS keeps record of such information in local cache memory (e.g., in a local hard disk). Based on this, the SBSs leverage the knowledge of past data requests so as to obtain recommendations on which UEs to service. In this regard, each SBS explores the records of past connections' MOSs using mathematical tools from collaborative filtering, which is one of the most widely-used concepts for building recommendation systems. In particular, collaborative filtering uses known users' information and correlations across similar services so as to make recommendations or predictions on which multimedia applications a new user is most likely to request [13], [14] and with which expected QoE. In summary, this work demonstrates how to exploit users' context information, available at each SBS, for making better informed decisions, 
thereby improving the overall network performance.

The remainder of this paper is organized as follows: Section II presents the considered system model and introduces the concepts of collaborative filtering for QoE prediction. In Section III, we formulate the UE-SBS association problem and present the proposed solution. In Section IV, we discuss the simulation results and, finally, conclusions are drawn in Section V.

\section{SySTEM MODEL}

Consider the downlink transmission of an orthogonal frequency division multiple access (OFDMA) small cell network. Let $\mathcal{M}=$ $\{1, \ldots, M\}$ and $\mathcal{N}=\{1, \ldots, N\}$ respectively denote the set of UEs and SBSs deployed in the network. Each SBS $i$ serves a set $\mathcal{L}_{i}$, up to a maximum quota $Q_{i}$. Each UE $m \in \mathcal{L}_{i}$ is assigned a bandwidth $w_{i, m}$ and the transmit power for the transmission to UE $m \in \mathcal{L}_{i}$ is $p_{i}$. The data rate achieved by UE $m \in \mathcal{L}_{i}$ is denoted by $r_{i, m}$.

Each UE $m$ uses a set $\mathcal{A}_{m}$ of data applications, whose data traffic generation is modeled as a stochastic process with source rate $s_{a, m}, a \in \mathcal{A}_{m}$. Since we focus on multimedia services, a suitable metric for the quality of service of these applications is given by the mean opinion score (MOS) [15] - a low complexity metric, which depends on the characteristics of the applications i.e., the source rate - and the wireless transmission - i.e., the packet error rate (PER). The MOS for a UE $m \in \mathcal{L}_{i}$ using application $a \in \mathcal{A}_{m}$ at a given instant $t$, is defined as [15] :

$$
\operatorname{MOS}_{i, m}^{a}(t)=\frac{c_{1}+c_{2} \log \left(s_{a, m}\right)}{1+c_{3} \mathrm{PER}_{i, m}+c_{4}\left(\mathrm{PER}_{i, m}\right)^{2}},
$$

where the values $\left\{c_{1}, \ldots, c_{4}\right\}$ are normalization parameters which bound the MOSs to the interval $[1,5]$. The packet error rate depends on the signal to interference and noise ratio (SINR), and for successful transmissions (i.e., to avoid packet error events), the SINR is required to be above a threshold $\Gamma$.

\section{A. MOS Prediction via Collaborative Filtering}

In SBS networks, UEs serviced by the same SBS for the same application are scheduled similarly and, on average, experience similar QoE. As a result, given a matrix with the MOSs from past application requests, an SBS can predict the MOSs for new UEs whose application requests are correlated with those in the matrix. Although this user information is easily obtainable by each SBS, over time, it can grow much larger and faster than the ability to process it for network optimization purposes. Therefore, the useful exploitation of user information is contingent upon efficiently searching and retrieving data from large and sparse databases, in a scalable way. For sifting through such large databases, we apply novel collaborative filtering (CF) techniques [13], [14]. In the proposed CF setting, each SBS $i$ maintains a matrix with the UEs $m \in \mathcal{L}_{i}$ and the applications $\mathcal{A}_{m}$ over time. Such knowledge can be represented in form of matrix as shown in Table I. The $a$-th column of Table I refers to the MOSs of different users who requested the same application $a$, while the $m$-th row encompasses the MOSs of user $m$ across his $\left|\mathcal{A}_{m}\right|$ data service requests. Finally, the dashes denote unavailable information, for service requests which still have not occurred. The goal of the CF approach is filling and updating the users' entries per application,
TABLE I

REPRESENTATION OF THE MOS MATRIX AT THE SBS.

\begin{tabular}{c||c|c|c|r} 
& $a=1$ & $a=2$ & $\ldots$ & $a=\left|\mathcal{A}_{m}\right|$ \\
\hline \hline$m=1$ & 5 & - & $\ldots$ & - \\
\hline$m=2$ & - & 4.3 & $\ldots$ & 2.5 \\
\hline$\ldots$ & $\ldots$ & $\ldots$ & $\ldots$ & $\ldots$ \\
\hline$m=\left|\mathcal{L}_{i}\right|$ & - & 2.6 & $\ldots$ & -
\end{tabular}

by exploiting the correlations among similar service requests (i.e., across columns), and similar user contexts (across rows). In this work, this information is used by the SBSs to predict the MOS of a given application request and locally devise the UE-SBS association which delivers the largest MOS.

In practice, when a new user arrives - e.g. UE $m$ using application $a \in \mathcal{A}_{m}$ - an SBS $i$ identifies two sets of data, called neighborhoods, which serves as baseline for predicting the MOS of UE $m$. The first neighborhood $\mathcal{S}_{a} \subseteq \mathcal{M}$ is composed by the MOSs of other UEs $n \neq m$ previously serviced by SBS $i$ for the same application $a$ (equivalent to a subset of the $a$-th column of Table I). The second neighborhood $\mathcal{S}_{m} \subseteq \mathcal{A}_{m}$ is defined by the MOSs of other applications in $\mathcal{A}_{m}$ previously requested by UE $m$ (subset of the $m$-th row of Table I). Finally, at SBS $i$, the prediction of the MOS of an UE $m$ requesting an application $a \in \mathcal{A}_{m}$ at time $t$, and willing to be associated to SBS $i$ can be expressed as [13] :

$\hat{x}_{i, m}^{a}(t)=\left(\bar{x}_{i}+b_{a}+b_{m}\right)+\frac{\sum_{n \in \mathcal{S}_{a}} d_{m, n} \hat{x}_{i, n}^{a}}{\sum_{n \in \mathcal{S}_{a}}\left|d_{m, n}\right|}+\frac{\sum_{\dot{a} \in \mathcal{S}_{m}} d_{a, \dot{a}} \hat{x}_{i, m}^{\dot{a}}}{\sum_{\dot{a} \in \mathcal{S}_{m}}\left|d_{a, \dot{a}}\right|}$,

where, $\bar{x}_{i}$ is the average MOS over all known UEs and applications; $b_{a}=\frac{\sum_{m \in \mathcal{S}_{a}} \hat{x}_{i, m}^{a}}{\left|\mathcal{S}_{a}\right|}-\bar{x}_{i}$ denotes the average MOS of the UEs connected to SBS $i$, using the same application $a$, relative to the average $\bar{x}_{i} ; b_{a}=\frac{\sum_{a \in \mathcal{S}_{m}} \hat{x}_{i, m}^{a}}{\left|\mathcal{S}_{m}\right|}-\bar{x}_{i}$ models the average MOS of UE $m$ over all the applications $\dot{a}$, with respect to the average $\bar{x}_{i}$. Finally, $d_{m, n}=\left|\hat{x}_{i, m}^{a}-\hat{x}_{i, n}^{a}\right|$ and $d_{a, \dot{a}}=\left|\hat{x}_{i, m}^{a}-\hat{x}_{i, m}^{\dot{a}}\right|$ respectively denote the absolute error (also referred to as distance) between the MOS of different UEs serviced by SBS $i$ (i.e., the neighborhood of application $a$ ), and different applications for a given UE $m$ (neighborhood of UE $m$ ). The nominal value of the unavailable entries (denoted by dashes in Table I) is set to zero.

Finally, a suitable function for capturing the MOS prediction accuracy and the UE-SBS association performance is given by the relative error between the actual MOS and the predicted one, defined as:

$$
U_{i, m}^{a}(t)=\frac{\left|\operatorname{MOS}_{i, m}^{a}(t)-\hat{x}_{i, m}^{a}(t)\right|}{\left|\operatorname{MOS}_{i, m}^{a}(t)\right|}
$$

For the cost function in (3), values close to zero represent optimal predictions, i.e., the best UE-SBS association for a given application $a$, and the prediction errors are less significant as long as the UE-SBS association guarantees large MOSs. Naturally, as such knowledge increases over time, sharpening the predictions, the proposed concept aims at optimizing the long-term UE-SBS association problem, rather than the myopic performance. 


\section{Cell Association as a Matching Game with EXTERNALITIES}

\section{A. Problem Formulation}

In this work, we aim at solving the problem of assigning each UE $m \in \mathcal{M}$ to the SBS $i \in \mathcal{N}$ that minimizes the cost function in (4) over a time horizon $T$, through a matching $\eta: \mathcal{M} \rightarrow \mathcal{N}$. Moreover, we attempt to bridge the gap between exploiting the correlations between similar application requests and providing differentiated QoE for the user's application requests. Essentially, this yields the following optimization problem:

$$
\begin{aligned}
& \underset{w_{i, m}, \hat{x}_{i, m}^{a}(t), \eta:(i, m) \in \eta}{\arg \min } \frac{1}{T} \sum_{t}^{T} \sum_{i \in \mathcal{N}} \sum_{m \in \mathcal{L}_{i}} \sum_{a \in \mathcal{A}_{m}} U_{i, m}^{a}(t) \\
& \text { s.t., }\left|\mathcal{L}_{i}\right| \leq Q_{i}, \forall i \in \mathcal{N}, \\
& \mathrm{r}_{i, m} \geq s_{a, m}, \forall m \in \mathcal{M}, i \in \mathcal{N}, \\
& \hat{x}_{i, m}^{a}(t), \operatorname{MOS}_{i, m}^{a}(t) \in[0,5], \forall m \in \mathcal{M}, i \in \mathcal{N},
\end{aligned}
$$

where (5) is a constraint on the maximum number of UEs that an SBS can service, constraint (6) represents a minimum data rate requirement, and (7) defines the domains of MOSs and CF-based estimations.

In terms of complexity, solving the UE-SBS association in (4) using classical optimization techniques is an NP-hard problem, which depends on the number of SBSs and UEs in the network. Even by relaxing some of the constraints in (5)-(7), the exponential complexity makes a centralized approach intractable, especially in SBS networks where the number of UEs and SBSs can significantly grow large.

This complexity coupled with the need for self-organizing solutions in small cells mandates a distributed approach in which UEs and SBSs autonomously decide on the best UE-SBS association. Accordingly, in order to solve the problem in (4), while avoiding combinatorial complexity, a suitable mathematical framework is given by matching games with externalities [16], [17]:

Definition 1. A matching game with externalities is defined by a tuple $(\mathcal{M}, \mathcal{N}, U)$, where $(\mathcal{M}, \mathcal{N})$ are the set of players, and a real valued function $U_{i, m}(t \mid \eta)$ that represents the cost associated to link $(i, m) \in \eta$ at time $t$, when matching $\eta$ forms.

The outcome of a matching game is a matching function (or association) $\eta$ that bilaterally assigns to each player $m \in \mathcal{M}$, a player $i=\eta(m), i \in \mathcal{N}$, and vice versa (i.e., $m=\eta(i)$ ). To reach a stable matching, each player $m \in \mathcal{M}, i \in \mathcal{N}$ uses relations $\succ_{m}, \succ_{i}$ so as to build preferences over one another, i.e., to rank, respectively, the players in $\mathcal{N}$ and $\mathcal{M}$. A preference relation $\succ$ is defined as a complete, reflexive, and transitive binary relation between the players in $\mathcal{M}$ and $\mathcal{N}$. Thus, for any UE $m$, a preference relation $\succ_{m}$ is defined over the set of SBSs $\mathcal{N}$ such that, at a given time instant, for any two SBSs $i, j \in \mathcal{N}^{2}, i \neq j$, and two matchings $\eta, \eta^{\prime} \in \mathcal{M} \times \mathcal{N}, i=\eta(m), j=\eta^{\prime}(m)$ :

$$
\begin{aligned}
& (i, \eta) \succ_{m}\left(j, \eta^{\prime}\right) \Leftrightarrow \\
& U_{i, m}^{a}(t \mid \eta)>U_{j, m}^{a}\left(t \mid \eta^{\prime}\right) .
\end{aligned}
$$

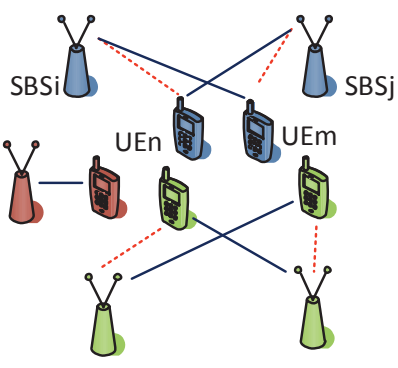

(a) Network scenario.

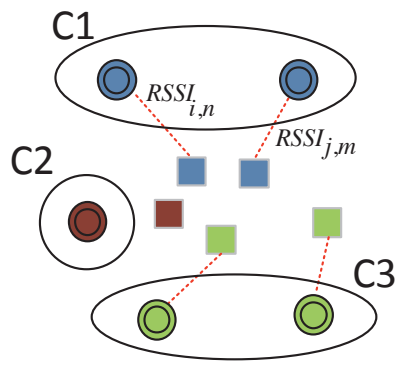

(b) Weighted interference graph.
Fig. 1. Construction of the interference graph. Each color indicates one operating frequency, blue lines denote useful signals, red dashed lines represent interference signals. Cliques are denoted by ovals. Note that, in the interference graph, the externalities (i.e., interference) only exist within each clique (e.g., $C 1, C 3$ ).

Similarly, for any SBS $i$ a preference relation $\succ_{i}$ over the set of UEs $\mathcal{M}$ is defined as follows, for any two UEs $m, n \in \mathcal{M}$, $m \neq n$ and two matchings $\eta, \eta^{\prime} \in \mathcal{M} \times \mathcal{N}, m=\eta(i), n=\eta^{\prime}(i)$ :

$$
\begin{aligned}
& (m, \eta) \succ_{i}\left(n, \eta^{\prime}\right) \Leftrightarrow \\
& U_{i, m}^{a}(t \mid \eta)>U_{i, n}^{a}\left(t \mid \eta^{\prime}\right) .
\end{aligned}
$$

By observing (8) and (9), one must note that the preference relations are a function of the existing matching $\eta$, at time $t$. In fact, since UE-SBS links composing the matching share the same pool of spectral resources, they affect each other's performance through co-channel interference. The interference across links indicates that, for the studied matching problem, the preferences of UEs and SBSs are interdependent, as they are both function of the PER. These interdependence relationships are known in matching theory as externalities, and have important implications in the design of the proposed solution, as we discuss in the following section.

\section{B. Context-aware UE-SBS association approach}

While most solution concepts for matching games, such as [6], [7], [18], assume that the preferences of a player do not depend on the other players' choices, this assumption does not hold for the considered UE-SBS association problem. As a matter of fact, when dealing with externalities, the potential link $(i, m)$ between an SBS $i$ and a UE $m$ depends on the other UE-SBS associations in $\eta \backslash(i, m)$. Consequently, traditional solution approaches based on preference orders, such as the deferred acceptance algorithm used in [7], [18], are now unsuitable as the ranking of the preference changes as the matching forms. Moreover, choosing greedy cost-minimizing preferences does not ensure matching stability. In fact, due to externalities, a player may continuously change its preference order, in response to the formation of other UE-SBS links, and never reach a final UE-SBS association, unless externalities are well-handled.

To manage the externalities across interfering UE-SBS links, we propose the representation of the initial SBSs network as an interference graph [19]. In such an approach, the network topology shown in Figure 1.a is translated into the interference graph of Figure 1.b, in which the vertices are interfering SBSs and UEs and the weight of the corresponding edges (shown as red dashed lines) is the received interference signal strength 


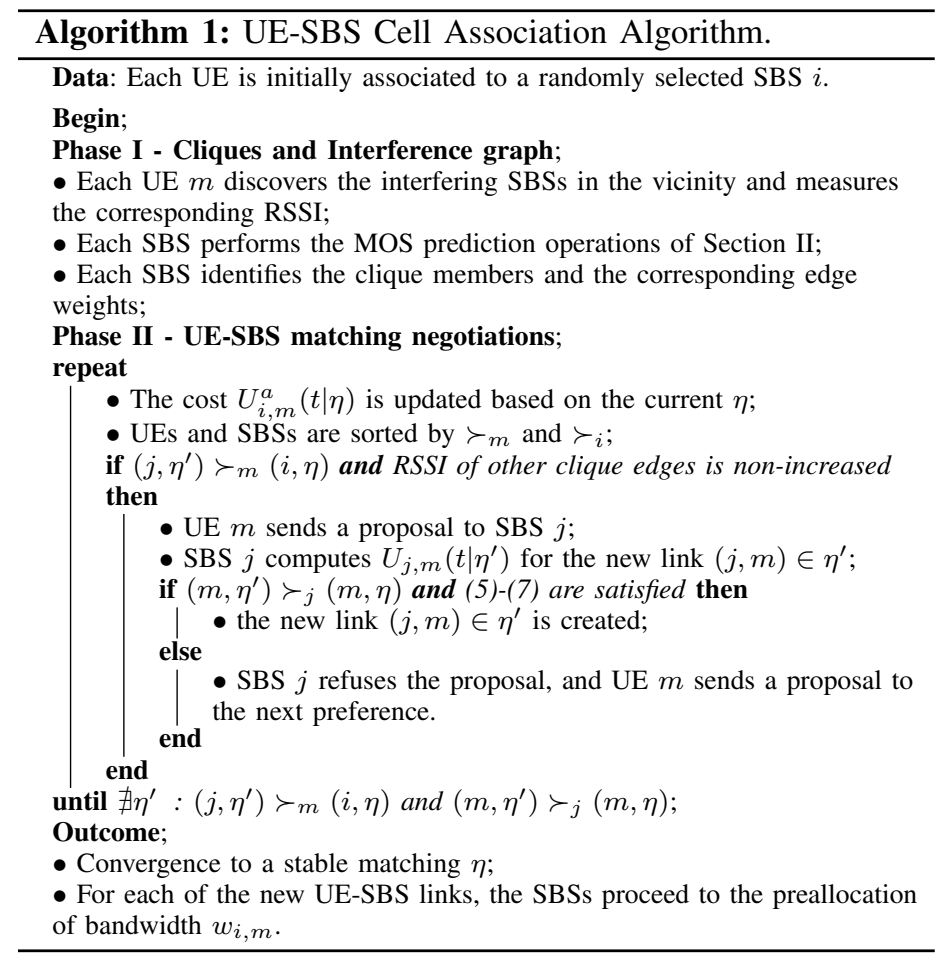

indicator (RSSI) at the UE $\operatorname{side}^{1}$. A graph-based representation allows to partition the original network into groups of mutually interfering SBS called cliques. The UE-SBS associations taking place within each clique compose a sub-matching, which is a subset of the larger matching defined over the entire network. A graph-based representation is particularly useful as it yields two important considerations. First, since the externalities only affect the members of the same clique (through the preferences in (8) and (9)), the UE-SBS association is a process that pertains to each distinct sub-matching. In other words, with refer to Figure 1.b., the formation of a sub-matching in clique $C 1$ is independent from the similar process in clique $C 3$, since no externalities exist across separate cliques. Second, the absence of cross-clique externalities allows to decompose the original matching problem, defined over the entire network, into clique-based sub-matching problems, defined over smaller clusters of interfering SBSs and UEs. In summary, introducing a graph-based partition of the network into cliques of interfering SBSs reflects a logical decomposition of the original problem into smaller ones, each with smaller number of players and reduced complexity.

Given of these considerations, we propose a decentralized solution to the problem in (4), shown in Algorithm 1. In the first phase of Algorithm 1, each SBS predicts the MOSs of the incoming $\mathrm{UEs}^{2}$ through the operation described in Section II . Also, in this phase, each SBS identifies the interfering SBSs and, based on that, it constructs a weighted interference graph of its belonging clique. In the second phase of Algorithm 1, each SBS

\footnotetext{
${ }^{1}$ Naturally, the proposed solution can also accommodate other equivalent interference indicators, such as the interference to noise ratio (INR).

${ }^{2}$ The target users can be identified based on their current location or by combining the proposed algorithm with mobility pattern prediction techniques [8].
}

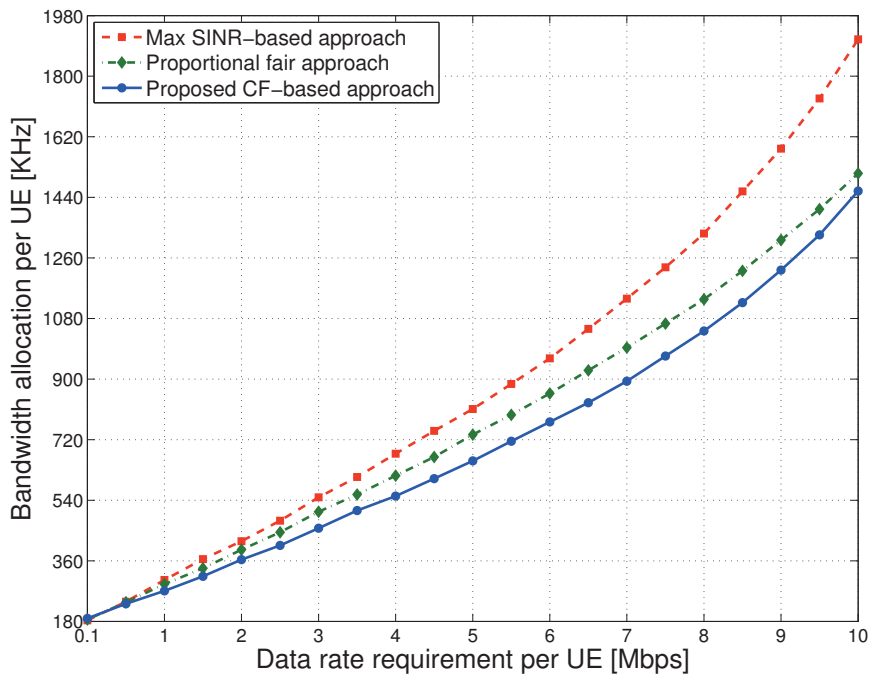

Fig. 2. Bandwidth allocation per UE as a function of the data rate requirement. $M=30$ UEs, $N=20$ SBSs

engages in mutual negotiations with other SBSs in the same clique for devising the UE-SBS association which minimizes the cost function as per (3), while accounting for the constraints (5)-(7). At the end of this stage, each UE is associated with the SBS which maximizes its MOS, or equivalently, best accommodates the UEs' application requests by minimizing the prediction error on its QoE requirements.

The matching stability follows from the lemma below:

Lemma 1. A matching $\eta$ is stable if, for each clique $C \subseteq \eta$, the creation of a new link $(i, m) \in C$ at a given instant $t$, verifies that $R S S I_{i, n}(t) \leq R S S I_{i, n}(t-1)$, for all UEs $n \in C$.

Proof. The proof follows from the preference relation definitions in (8) and (9). Since the preference relations of UE $n$ and SBS $j$ are functions of the received interference (through the $P E R_{j, n}$ ), a sufficient condition for the stability of a sub-matching (i.e., the matching within a clique) is that the formation of any new UESBS link $(i, m)$ does not undermine the stability of the existing ones. Such a condition is verified if the interference received by any UE $n$ in clique $C$ does not increase after the formation of link $(i, n)$, as denoted by $R S S I_{i, n}(t) \leq R S S I_{i, n}(t-1)$. In fact, in this case, the preference orders of all UEs $n$ and SBSs $j$ in the clique remain unaltered after the creation of link $(i, m)$, which ensures the stability of the sub-matching, defined over such clique. Finally, since no externalities exist across cliques, the stability of the network-wide matching follows from the stability of the submatchings composing it.

\section{Simulation RESUlts}

For numerical simulations, we consider a single macro-cell with a $1 \mathrm{~km}$ radius and a bandwidth of $10 \mathrm{MHz}$. In this cell, $M$ UEs and $N$ SBSs are uniformly deployed. The transmit power of each SBS $i$ is set to $30 \mathrm{dBm}$. Transmissions are affected by distance dependent path loss and shadowing according to 3GPP specifications [2]. The minimum bandwidth assignment $w_{i, m}=180 \mathrm{KHz}$, and each SBS $i$ can service up to $Q_{i}=6$ 


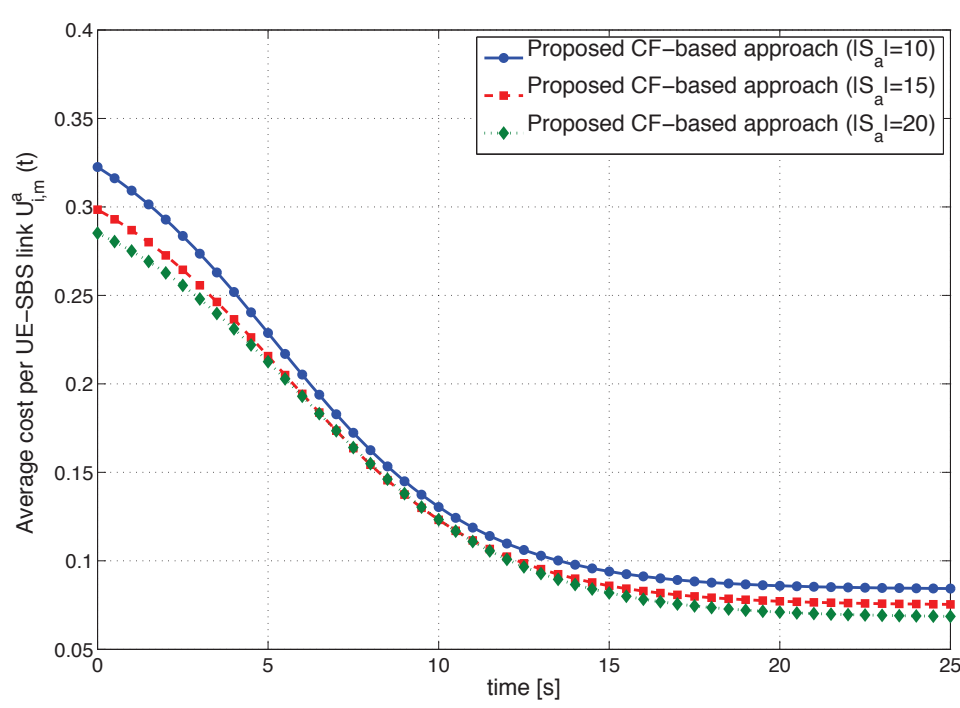

Fig. 3. Timelapse of the average cost per UE-SBS link in the proposed approach, for different neighborhood size $\left|S_{a}\right|=\{15,20,25\} .\left|S_{m}\right|=2, M=40$ UEs, $N=20$ SBSs.

UEs [2]. A packet error event is the result of a SINR at each UE smaller than $\Gamma=9.56 \mathrm{~dB}$ [2], the noise level is $\sigma^{2}=-121 \mathrm{dBm}$.

For comparison purposes, we also considered two baseline solutions for user cell association [3]. In the first scheme, the UE is associated to the SBS providing the largest SINR. In the second scheme, each UE is associated to the SBS that maximizes the ratio between instantaneous and average rate. Specifically, in this latter scheme, the serving SBS is the one that, for a given bandwidth allocation maximizes the ratio between instantaneous and average data rate, based on a proportional fair (PF) scheduler. Clearly, the above schemes are not proactive, hence the SBSs allocate the spectral resources upon receiving the UEs' application requests.

In Figure 2, we show the average bandwidth allocation per $\mathrm{UE}$ as a function of the data rate requirement, in a network with $M=60$ UEs, using $\left|\mathcal{A}_{m}\right|=3$ applications. Figure 2 shows that leveraging UE context information allows for more precise spectrum allocations, ultimately yielding $19 \%$ spectrum savings with respect to the max-SINR approach and $10.1 \%$ with respect to $\mathrm{PF}$, for a target data rate of $7 \mathrm{Mbps}$.

In Figure 3, we observe the time evolution of the average cost per UE-SBS link for all the considered approaches. Figure 3 shows that the accuracy of the proposed approach increases over time and when larger datasets are available ( equivalently, when the CF prediction is based on large neighborhood sizes $\left|S_{m}\right|$ ). For instance, the proposed approach achieves a MOS prediction error $U_{i, m}^{a} \leq 10 \%$, after a simulation time of $14 \mathrm{~s}$, with a scheduling period of $0.5 \mathrm{~s}$, in a network composed by $N=20 \mathrm{SBSs}$ and $M=40$ UEs. In summary, Figure 3 demonstrates that the proposed algorithm achieves good predictions with a reasonable initial delay, even in network of regular dimensions.

\section{CONCLUSIONS}

In this paper, we have presented a novel approach to the cell association problem in small cell networks, which exploits the user context information and a QoE prediction. The proposed scheme brings forward the important advantage of providing a UE-SBS association which can deliver high and differentiated QoE, based on the distributed knowledge of practical context information extracted from modern UE devices. We have modeled the UESBS association problem as a matching game with externalities, in which the UEs and SBSs build preferences over one another so as to choose their preferred matching. Given that the preferences are interdependent and a function of the existing matching, we have proposed an interference graph-based algorithm that decomposes the original problem into smaller ones, defined over clique of interfering SBSs, while accounting for the network externalities. Simulation results have shown that the proposed approach can provide significant gains in terms of spectrum savings for given data rate targets, with an acceptable initial delay, reaching up to $19 \%$, with respect to a traditional context-unaware SBS-UE association which is based on the maximum SINR.

\section{REFERENCES}

[1] H.-S. Jo, Y. J. Sang, P. Xia, and J. G. Andrews, "Heterogeneous cellular networks with flexible cell association: A comprehensive downlink SINR analysis," IEEE Transactions on Wireless Communications, vol. 11, no. 10, pp. 3484-3495, 2012

[2] W. C. Cheung, T. Quek, and M. Kountouris, "Access control and cell association in two-tier femtocell networks," in In Proc. of IEEE Int'l Wireless Comm. and Networking Conference (WCNC), Apr. 2012.

[3] T. Q. S. Quek, G. de la Roche, I. Guvenc, and M. Kountouris, Small Cell Networks: Deployment, PHY Techniques, and Resource Management. New York, USA: Cambridge University Press, Sept. 2012.

[4] J. Andrews, H. Claussen, M. Dohler, S. Rangan, and M. Reed, "Femtocells: Past, present, and future," IEEE Journal on Sel. Areas in Comm., vol. 30, no. 3, pp. $497-508$, Apr. 2012.

[5] R. Madan, J. Borran, A. Sampath, N. Bhushan, A. Khandekar, and T. Ji, "Cell association and interference coordination in heterogeneous LTE-A cellular networks," IEEE Journal on Sel. Areas in Comm., vol. 28, no. 9, pp. $1479-1489$, Dec. 2010.

[6] L. Jiang, S. Parekh, and J. Walrand, "Base station association game in multicell wireless networks," in In Proc. of IEEE Wireless Communications and Networking Conference, Apr. 2008, pp. 1616 -1621.

[7] A. Leshem, E. Zehavi, and Y. Yaffe, "Multichannel opportunistic carrier sensing for stable channel access control in cognitive radio systems," IEEE J. Select. Areas Commun., vol. 30, no. 1, pp. 82-95, Jan. 2012.

[8] W. N. Schilit, A system architecture for context-aware mobile computing New York, USA: PhD Thesis, Columbia University, 1995.

[9] G. Chen and D. Kotz, "A survey of context-aware mobile computing research," Hanover, NH, USA, Tech. Rep., 2000.

[10] E. Bastug, J.-L. Guenego, and M. Debbah, "Proactive small cell networks," in In Proc. of Int'l Conf. on Telecommunications (ICT), 2013, 2013, pp. 1-5.

[11] N. Golrezaei, K. Shanmugam, A. Dimakis, A. Molisch, and G. Caire, "Femtocaching: Wireless video content delivery through distributed caching helpers," in In Proc. of IEEE INFOCOM, 2012, pp. 1107-1115.

[12] H. Ahlehagh and S. Dey, "Adaptive bit rate capable video caching and scheduling," in In Proc. of Wireless Communications and Networking Conference, 2013, pp. 1357-1362.

[13] X. Su and T. M. Khoshgoftaar, "A survey of collaborative filtering techniques," Advances in Artificial Intelligence, vol. 09, no. 10, pp. 1-19, 2009.

[14] B. Sarwar, G. Karypis, J. Konstan, and J. Riedl, "Item-based collaborative filtering recommendation algorithms," in In Proc. of the 10th International Conference on World Wide Web, Hong Kong, 2001, pp. 285-295. [Online]. Available: http://doi.acm.org/10.1145/371920.372071

[15] J. Xu, L. Xing, A. Perkis, and Y. Jiang, "On the properties of mean opinion scores for quality of experience management," in In Proc. of Int'l Symp. on Multimedia (ISM), 2011, pp. 500-505.

[16] A. Roth and M. A. O. Sotomayor, Two-Sided Matching: A Study in GameTheoretic Modeling and Analysis. New York, USA: Cambridge Press, 1992.

[17] S. Brânzei, T. P. Michalak, T. Rahwan, K. Larson, and N. R. Jennings, "Matching games with additive externalities," CoRR, vol. abs/1207.3682, 2012.

[18] Y. Wu, H. Viswanathan, T. Klein, M. Haner, and R. Calderbank, "Capacity optimization in networks with heterogeneous radio access technologies," in In Proc. of IEEE Globecom 2011,.

[19] B. Balasundaram and S. Butenko, Graph Domination, Coloring and Cliques in Telecommunications. New York, USA: Springer, 2006. 